\title{
Tecnologías no convencionales para la remoción de plomo presente en aguas residuales: una revisión bibliográfica 2010-2019
}

\section{Unconventional technologies for the removal of lead present in}

wastewater: a 2010-2019 bibliographical review

\author{
Dora Luz Gómez Aguilar ${ }^{10}$, Javier Andrés Esteban Muñoz ${ }^{2}{ }^{(0)}$, \\ Deisy Baracaldo Guzmán ${ }^{3}$
}

Fecha de recepción: 01 de octubre de 2019 Fecha de aceptación: 05 de Febrero de 2020

Cómo citar: Gómez-Aguilar, D.L., Esteban-Muñoz, J.A. y Baracaldo-Guzmán, D. (2020). Tecnologías no convencionales para la remoción de plomo presente en aguas residuales: una revisión bibliográfica 2010-2019. Tecnura, 24(64). 97-116 https://doi.org/10.14483/22487638.15849

\section{RESUMEN}

Objetivo: El presente artículo de revisión tuvo como objetivo realizar una indagación bibliográfica de artículos científicos en revistas locales, nacionales e internacionales en el periodo de 2010 a 2019, con relación a las tecnologías no convencionales (biopolímeros, biorremediación y residuos agroindustriales) que se han empleado en la remoción de plomo $(\mathrm{Pb})$, presente en aguas residuales sintéticas o industriales.

Metodología: La investigación adoptó un modelo investigativo de corte cualitativo, de tipo exploratorio. Los aspectos metodológicos correspondieron a la indagación y elección de artículos científicos (para cada una de las tecnologías no convencionales seleccionadas) del periodo 2010-2019, teniendo como criterio palabras clave; posteriormente, se realizó un análisis de esos contenidos y se diligenciaron dos matrices realizadas en Excel.

Resultados: La revisión bibliográfica permitió observar que: en primer lugar, con relación a las tecnologías no convencionales con mayor tendencia en investigación, empleadas en la remoción de plomo, se encuentran los residuos agroindustriales, seguidos por la biorremediación y por último los biopolímeros, para lo cual se hal-

Licenciada en Química, Magíster en Biología con énfasis en Fitoquímica, Doctora en Desarrollo Sostenible, Docente de la Universidad Pedagógica Nacional. Bogotá, Colombia. Contacto: dgomez@pedagogica.edu.co. ORCID: https://orcid.org/0000-0002-5722-9063

2 Tecnólogo en Química Aplicada a la Industria. Tecnólogo en Electricidad Industrial. Licenciado en Química. Candidato a Magíster en Docencia de la Química, Maestrante de la Universidad Pedagógica Nacional. Bogotá, Colombia. Contacto: dqu_aestebanm214@pedagogica.edu.co ORCID: https://orcid.org/0000-0003-1158-4091

Magíster en Tecnologías de la Información Aplicada a la Educación. Universidad Pedagógica Nacional. Bogotá, Colombia. Contacto: dbaracaldo@pedagogica.edu.co.

ORCID: https://orcid.org/0000-0002-0866-6822 
laron y seleccionaron 19 para el primero, 13 para el segundo y 9 para el último. De igual forma, los países que han reportado resultados investigativos alrededor de las tecnologías no convencionales seleccionadas, corresponden a: China, Colombia, Corea, Egipto, España, Francia, India, Indonesia, Irán, Nigeria, Malasia, México, Perú, Romania, Serbia, Singapur y Zimbabue; en contraste, las publicaciones seleccionadas se encuentran en su gran mayoría ubicadas a nivel internacional. Por último, los porcentajes de remoción más alto del orden del $100 \%$ se presentaron con los residuos agroindustriales (para lo cual se han investigado 24); seguido del $98 \%$, con la biorremediación (donde se han investigado algas rojas y seis cepas de bacterias), y finalmente, los biopolímeros, con 95,32 \% (donde el quitosano, el almidón, la queratina, las conchas de caracol y las plumas, se han investigado).

Conclusiones: Las tecnologías no convencionales con mayor tendencia en investigación y aplicación en matrices de aguas residuales industriales, de acuerdo con periodo 2010-2019, corresponden a los residuos agroindustriales; de igual forma, la mayoría de aguas residuales en las que han sido aplicados estos métodos corresponde a las sintéticas, lo que conlleva a exponer que estas deben comenzar a ser implementadas a nivel industrial o un pilotaje, para observar su eficiencia en la remoción de metales pesados, y de forma particular el plomo.

Financiamiento: La investigación llevada a cabo no recibió financiación.

Palabras clave: aguas residuales; plomo; remoción; tecnologías no convencionales.

\section{ABSTRACT}

Objective: The objective of this review article was to carry out a bibliographic inquiry at the level of scientific articles in local, national and international journals in the period from 2010 to 2019, in relation to unconventional technologies (biopolymers, bioremediation, and agroindustrial waste) that they have been used in the removal of lead $(\mathrm{Pb})$, present in synthetic and / or industrial wastewater.

Methodology: The research adopted a qualitative, investigative model of exploratory type. The methodological aspects carried out corresponded to the inquiry and choice of scientific articles (for each of the unconventional technologies selected) in the period from 2010 to 2019, based on keywords; subsequently, an analysis of their content was carried out and two matrices performed in Excel were filled out.

Results: The bibliographic review carried out allowed us to observe that: firstly, in relation to the unconventional technologies with the greatest tendency in research, used in the removal of lead, agroindustrial residues are found, followed by bioremediation and finally biopolymers, for which they found and selected 19 for the first, 13 for the second, and for the last nine. Similarly, the countries that have carried out and reported research results around the selected unconventional technologies correspond to: China, Colombia, Korea, Egypt, Spain, France, India, Indonesia, Iran, Nigeria, Malaysia, Mexico, Peru, Romania, Serbia, Singapore and Zimbabwe; in contrast, the selected publications are mostly located internationally. Finally, the highest removal percentages of the order of $100 \%$ were presented with agroindustrial wastes (for which 24 have been investigated), followed by 98\% with bioremediation (where red algae and six strains of bacteria have been investigated) and finally, biopolymers, with $95.32 \%$ (where chitosan, starch, keratin, snail shells and feathers, have been investigated).

Conclusions: The unconventional technologies with greater tendency in research and application in industrial wastewater matrices, ac- 
cording to the 2010-2019 period, correspond to agroindustrial waste; In the same way, the majority of wastewater in which these types of methods have been applied correspond to the synthetic ones, which leads to exposing that these must begin to be implemented at an industrial level or a pilot, to observe their ef- ficiency in the removal of heavy metals, and particularly lead.

Financing: The investigation carried out did not receive funding.

Keywords: Wastewater; lead; removal; unconventional technologies.

\section{INTRODUCCIÓN}

Uno de los retos del siglo XXI es mitigar el impacto ambiental producido por los metales pesados provenientes de actividades antropogénicas de sectores industriales, como: explotaciones mineras, fotografía, cerámicas, pinturas, galvanoplastia, electrónica y curtiembres, entre otras (León, Córdoba y Carreño, 2016; Gómez et al., 2019). Esto se debe a que dichos contaminantes inorgánicos no se biodegradan, se bioacumulan y se biomagnifican a lo largo de la cadena trófica, trayendo impactos negativos al ecosistema (Londoño-Franco, Londoño-Muñoz y Muñoz-García, 2016).

De acuerdo con lo anterior, según la Organización Mundial de la Salud (OMS, 2011) son trece los metales pesados con mayor incidencia en la salud y en el ambiente, entre los cuales se encuentran: $\mathrm{Hg}$, $\mathrm{Pb}, \mathrm{Cr}, \mathrm{Mn}, \mathrm{Zn}, \mathrm{Cu}, \mathrm{Co}, \mathrm{Ti}, \mathrm{As}, \mathrm{Cd}, \mathrm{Ni}$, Sn y Fe. Por esta razón, universidades como Columbia y Yale, desde 1999, han desarrollado sistemas de evaluación de desempeño ambiental, entre los cuales se encuentra el indicador de desempeño ambiental (EPI, por su sigla en inglés), el cual permite medir las políticas ambientales de 180 países, los cuales deben relacionarse con las metas propuestas de los Objetivos del Desarrol- lo Sostenible, promulgados en la Agenda 2030 (Ojeda, Spoor y Estrada, 2017).

Por esta razón, los metales pesados se han convertido de uno de los 24 indicadores del EPI, que forman parte del objetivo y de la política de salud ambiental; de ellos, el único metal que se tiene referenciado por su alto grado de toxicidad y por las repercusiones en la salud, en contraste con los trece catalogados por la OMS, corresponde al plomo. Esto se debe a que, dicho contaminante inorgánico ha sido clasificado como una amenaza ambiental (por su prevalencia en el agua, suelo y aire) y la salud de mujeres embarazadas y niños (Wendling et al., 2018), y la medición en la exposición a este metal se emplea el número de años de vida ajustados por discapacidad estandarizados por edad (AVAD) que se pierden por cada 100000 personas, debido a este riesgo. Teniendo en cuenta este último aspecto, y lo mencionado anteriormente, la población infantil es sensible a esta sustancia, dado que puede absorber de cuatro a cinco veces más que un adulto, lo cual puede ocasionar problemas cognitivos como retraso metal, dificultades de aprendizaje, trastornos de conducta, anemia, daño en los riñones y el cerebro, debilidad muscular. Para el caso de las mujeres en etapa de embarazo, puede llevar a malformaciones fetales, nacimientos prematuros e, incluso, 
a producir abortos espontáneos (Abadin et al., 2007; Wendling et al., 2018).

El plomo $(\mathrm{Pb})$ es un metal de número atómico 82 , masa atómica $207,20 \mathrm{~g} / \mathrm{mol}$, ubicado en el grupo 14, el cual tiene 38 isotopos, 2 estados de oxidación usuales, $2+$ y $4+$ y 514 minerales a nivel de la corteza terrestre que contienen este elemento en su composición química (Barthelmy, 2014). A nivel de sus aplicaciones se encuentran: baterías, metalurgia, industria farmacéutica, pigmentos, tuberías (ATSDR, 2007).

En cuanto a las repercusiones a la salud que puede ocasionar una exposición prolongada al plomo se encuentran: daño en los riñones, el sistema nervioso central (SNC) y el esperma, hemorragias retinianas y neuritis del nervio óptico, abortos espontáneos, y efectos negativos sobre el aprendizaje y el comportamiento en los niños (ATSDR, 2007; Abadin et al., 2007). En contraste, una de las enfermedades profesionales desarrollada por este metal, y que en el contexto de Colombia se encuentra contemplada en el Decreto 2566 de 2009, corresponde al saturnismo.

Con relación a los límites máximos permisibles (LMP - mg/L Pb), para el caso de las aguas residuales, se tienen establecidos particularmente para las industrias de curtiembres a nivel de algunos países del continente sur americano, así: Guayaquil y Guatemala $(0,2)$ (Legislación Secundaria del Ministerio de Ambiente de Guayaquil, 2004; Ministerio de Ambiente y Recursos Naturales de Guatemala, 2005); Perú $(0,5)$ (Sedapal, 2009); para Colombia, Venezuela y Brasil no se contempla en las regulaciones correspondientes.

Para el caso de EE. UU., el LMP es de 0,10 $\mathrm{mg} / \mathrm{L} \mathrm{Pb}$ (U.S. Environmental Protection
Agency, 2014); en el continente europeo, Finlandia, Grecia e Indonesia no lo contemplan en sus regulaciones, a diferencia de Madrid y Bangladesh que exponen un LMP de 1,0 y 0,1, respectivamente (Consejería de Presidencia y Portavocía del Gobierno Comunidad de Madrid, 2005; UNEP, 1999).

En complemento, atendiendo al EPI, cabe indicar que de acuerdo con la métrica de 2018 dada por la Universidad de Yale, los países que lideran el manejo adecuado de la contaminación producida por el plomo están: Suecia, Alemania, Japón y Finlandia; esto se debe a que han mejorado las regulaciones y mecanismos de monitoreo en la salud humana y ambiental, a diferencia de países como Afganistán, Haití, Pakistán y Bangladesh, que se encuentran catalogados como rezagados en la exposición a $\mathrm{Pb}$, dado que en sus actividades industriales continúan empleando este metal, sin implementar políticas rigurosas que conlleven al aseguramiento de la salud de las personas expuestas, así como el manejo de los residuos generados y que incorporan dicho metal (Wendling et al., 2018).

Por otra parte, entre los métodos de remoción reportados para el $\mathrm{Pb}$ se encuentran las tecnologías convencionales, como: adsorción con carbón activado, precipitación química (en soluciones alcalinas, precipitando como $\mathrm{Pb}(\mathrm{OH}) 4)$ y coagulantes inorgánicos. De igual forma, las tecnologías avanzadas empleadas son: filtración por membrana, ultrafiltración, microfiltración, electrodiálisis, electrocoagulación, ósmosis inversa, intercambio iónico y cementación (Caviedes et al., 2015; García V., García U. de Plaza, 2016). Estas dos técnicas, aunque son muy eficientes, presentan también ciertas desventajas como: alto costo en su 
implementación y mantenimiento en sistemas de tratamiento de aguas residuales (STAR), generan una cantidad considerable de lodos contaminados con el metal removido y, a su vez, no son asequibles económicamente para las diferentes industrias que empleen este metal en sus procesos.

Por esta razón, la presente investigación se enfocó en realizar una revisión bibliográfica entre el periodo de 2010 a 2019, con relación a las tecnologías no convencionales (también conocidas como verdes o limpias, (Amaringo y Hormaza, 2018), que se han empleado para la remoción de $\mathrm{Pb}$ (II), presente en aguas residuales sintéticas o industriales. El interés de los autores por indagar estas técnicas se debe a que, en comparación con los métodos anteriormente mencionados, son de fácil adquisición, implementación y mantenimiento en los STAR, no generan un volumen de lodo considerable y a su vez son biodegradables, lo cual permite que sean reutilizados como compostaje; entre los métodos no convencionales indagados se encuentran: biopolímeros, biorremediación y residuos agroindustriales; esto con el fin de analizar su aplicación en matrices reales.

Tabla 1. Biopolímeros empleados en la remoción de plomo presentes en aguas residuales (2010-2019)

\begin{tabular}{|c|c|c|c|c|c|c|}
\hline Año & Revista & País & Autor & $\begin{array}{l}\text { Tipo de bio } \\
\text { polímero }\end{array}$ & Fuente & Ref. \\
\hline 2019 & $\begin{array}{l}\text { Royal Society of } \\
\text { Chemistry }\end{array}$ & India & $\begin{array}{c}\text { Manzoor K, Ahmad M, Ahmad S, } \\
\text { Ikram S }\end{array}$ & $\begin{array}{l}\text { Quitosano } \\
\text { modificado }\end{array}$ & $\begin{array}{l}\text { Manzoor } \\
\text { et.al. (2019) }\end{array}$ & [4] \\
\hline 2019 & $\begin{array}{l}\text { AIP Conference } \\
\text { Proceedings }\end{array}$ & Malasia & $\begin{array}{l}\text { Nurul Huda Baharuddin, Nik } \\
\text { Meriam Nik Sulaiman, Mohamed } \\
\text { Kheireddine Aroua, Mohd Ghazali } \\
\text { Mohd Nawawi, Mohd Azraai } \\
\text { Kassim, Mohd Roslee Othman, y } \\
\text { Irvan Dahlan }\end{array}$ & Almidón & $\begin{array}{l}\text { Baharuddin } \\
\text { et al. (2019) }\end{array}$ & [1] \\
\hline 2019 & $\begin{array}{l}\text { Sustainable Environ- } \\
\text { ment Research }\end{array}$ & Morocoo & $\begin{array}{c}\text { Ablouh E, Hanani Z, Eladlani N, } \\
\text { Rhazi M, Taourirte M }\end{array}$ & $\begin{array}{l}\text { Microesferas } \\
\text { de quitosano } \\
\text { con alginato de } \\
\text { sodio } \\
\end{array}$ & $\begin{array}{l}\text { Ablouh et al. } \\
\qquad(2019)\end{array}$ & [7] \\
\hline 2014 & $\begin{array}{l}\text { Chemical Engineer- } \\
\text { ing Journal }\end{array}$ & China & $\begin{array}{c}\text { Kong J, Yue Q, Sun S, Gao B, Kan Y, } \\
\text { Li Q, Wang Y }\end{array}$ & $\begin{array}{l}\text { Residuos de } \\
\text { Queratina }\end{array}$ & $\begin{array}{l}\text { Kong J et al. } \\
(2014)\end{array}$ & [5] \\
\hline 2016 & $\begin{array}{l}\text { Environmental Tech- } \\
\text { nology \& Innovation }\end{array}$ & India & Anantha R, Kota, S. & $\begin{array}{l}\text { Plumas } \\
\text { Dromaius } \\
\text { novaehollan- } \\
\text { diae (DNF) y } \\
\text { Quitosano }\end{array}$ & $\begin{array}{l}\text { Anantha y } \\
\text { Kota }(2016)\end{array}$ & [8] \\
\hline 2016 & Scientific Reports & China & $\begin{array}{c}\text { Li B, Zhou F, Huang K, Wang Y, Mei } \\
\text { S, Zhou Y, Jing T }\end{array}$ & Polietilenimina & $\begin{array}{l}\text { Li et al. } \\
\text { (2016) }\end{array}$ & [9] \\
\hline 2012 & $\begin{array}{l}\text { 2nd International } \\
\text { Conference on } \\
\text { Environment and } \\
\text { BioScience }\end{array}$ & Singapur & $\begin{array}{l}\text { Michelle Castañeda, Ma. Stalin Mira- } \\
\text { sol, Lora Alaine Raymundo, Judilyn } \\
\text { Solidum }\end{array}$ & $\begin{array}{l}\text { Conchas de } \\
\text { caracol de } \\
\text { agua dulce } \\
\text { Melanoides } \\
\text { tuberculata } \\
\text { Muller (Family } \\
\text { Thiaridae) }\end{array}$ & $\begin{array}{l}\text { Castañeda et } \\
\text { al. (2012) }\end{array}$ & {$[2]$} \\
\hline 2010 & $\begin{array}{l}\text { African Journal of } \\
\text { Biotechnology }\end{array}$ & Nigeria & $\begin{array}{c}\text { Bamgbose J. T, Adewuyi S, Bamg- } \\
\text { bose O. y Adetoye A. A. }\end{array}$ & Quitosano & $\begin{array}{l}\text { Bambose et } \\
\text { al. (2010) }\end{array}$ & [3] \\
\hline 2010 & $\begin{array}{l}\text { Journal Environmen- } \\
\text { tal Sciences }\end{array}$ & Malasia & Wan Ngah W, Fatinathan S & $\begin{array}{l}\text { Quitosano y } \\
\text { derivados }\end{array}$ & $\begin{array}{l}\text { Wan Ngah } \\
\text { y Fatinathan } \\
(2010)\end{array}$ & [6] \\
\hline
\end{tabular}

Fuente: elaboración propia. 
Tabla 2. Variables óptimas de remoción para plomo presente en aguas residuales empleando biopolímeros

\begin{tabular}{|c|c|c|c|c|c|c|c|c|c|c|c|c|c|c|c|}
\hline 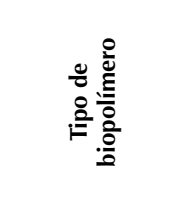 & 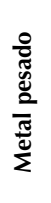 & 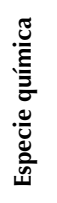 & 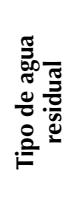 & 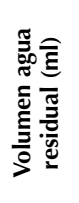 & 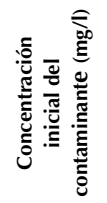 & 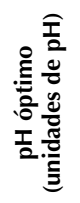 & $\begin{array}{l}\stackrel{U}{0} \\
\frac{T}{2}\end{array}$ & 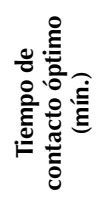 & 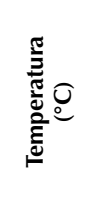 & 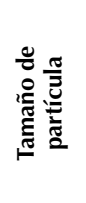 & 咅 & 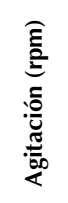 & 总 & 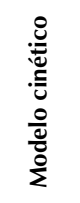 & 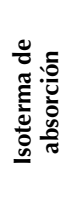 \\
\hline $\begin{array}{l}\text { Almidón no } \\
\text { modificado }\end{array}$ & $\mathrm{Pb}$ & II & 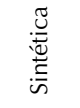 & 250 & 10 & $\begin{array}{c}2,0 \mathrm{a} \\
5,0\end{array}$ & NA & $\mathrm{NE}$ & 26 & $N E$ & 0,5 & NE & NA & NE & $\mathrm{NE}$ \\
\hline $\begin{array}{c}\text { Conchas de } \\
\text { caracol }\end{array}$ & $\mathrm{Pb}$ & II & 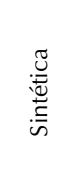 & 100 & 2 & 5,5 & NA & 480 & $\begin{array}{c}27 a \\
30\end{array}$ & $\begin{array}{l}0,25 \\
\mathrm{~mm}\end{array}$ & 50 & NE & NA & 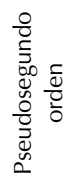 & NA \\
\hline Quitosano & $\mathrm{Pb}$ & II & 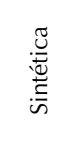 & 25 & $\begin{array}{l}100 \mathrm{a} \\
1000\end{array}$ & 4,5 & NA & NE & 25 & $\begin{array}{c}0,35 \\
- \\
0,5 \\
\mathrm{~mm}\end{array}$ & 4 & 180 & NA & $\mathrm{NE}$ & 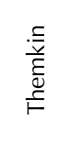 \\
\hline $\begin{array}{l}\text { Quitosano } \\
\text { modificado }\end{array}$ & $\mathrm{Pb}$ & II & 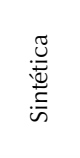 & NE & 50 & 6,5 & NA & 60 & 42 & NE & $\mathrm{NE}$ & NE & 己 & 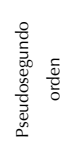 & 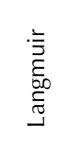 \\
\hline $\begin{array}{l}\text { Residuos de } \\
\text { queratina }\end{array}$ & $\mathrm{Pb}$ & II & 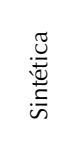 & NE & $\begin{array}{l}25- \\
150\end{array}$ & 5,5 & NA & $\mathrm{NE}$ & $\mathrm{NE}$ & $\begin{array}{c}50 \\
-60 \\
\text { malla }\end{array}$ & 1 & 160 & ర己 & 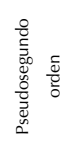 & 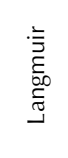 \\
\hline $\begin{array}{l}\text { Quitosano y } \\
\text { derivados }\end{array}$ & $\mathrm{Pb}$ & II & 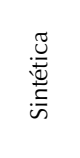 & 50 & 10 & 4,5 & 7,29 & 100 & $\mathrm{NE}$ & $\begin{array}{l}0,3269 \\
\text { m2/g } \\
\text { Sup. }\end{array}$ & 1 & 400 & 己े & $\mathrm{NE}$ & 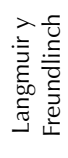 \\
\hline $\begin{array}{l}\text { Quitosano } \\
\text { modificado }\end{array}$ & $\mathrm{Pb}$ & II & 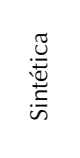 & NE & 300 & 3 a 5 & 6,6 & NE & $20-55$ & área & NE & NE & U. & $\mathrm{NE}$ & 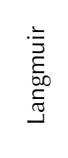 \\
\hline & $\mathrm{Pb}$ & II & 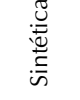 & 50 & 20 & 4,0 & NA & 70 & 30 & NE & 6 & 180 & & & \\
\hline $\begin{array}{c}\text { Plumas } \\
\text { Dromaius } \\
\text { novaehollandiae } \\
\text { (DNF) y } \\
\text { Quitosano }\end{array}$ & $\mathrm{Pb}$ & & 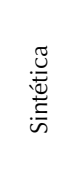 & & & & NA & & & & & & ป。 & 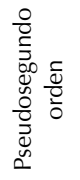 & 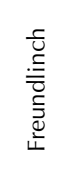 \\
\hline $\begin{array}{l}\text { Gelatina con } \\
\text { Polietilenimina }\end{array}$ & $\mathrm{Pb}$ & II & 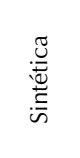 & 20 & 10 a 50 & 6,0 & NA & 720 & NE & $N E$ & 10 & 200 & 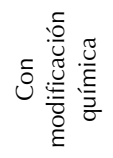 & 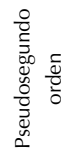 & 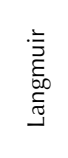 \\
\hline \multicolumn{16}{|l|}{$\begin{array}{l}\text { *NA: no aplica } \\
{ }^{*} \text { NE: no específi }\end{array}$} \\
\hline
\end{tabular}

Fuente: elaboración propia. 
Metodología: La investigación Ilevada a cabo fue de tipo exploratoria-cualitativa, dado que los autores realizaron una revisión bibliográfica.

Los criterios de búsqueda de artículos tuvieron presentes las siguientes palabras clave: tecnología no convencional, plomo, absorción, remoción, biopolímeros, biorremediación y residuos agroindustriales. Posteriormente, se elaboraron dos matrices en Excel para analizar los contenidos de acuerdo con los escritos seleccionados; para la primera matriz, se observó año del artículo, revista, país, autor(es), tipo de tecnología no convencional, fuente bibliográfica; por último, para la segunda matriz, los aspectos a analizar correspondieron a: tipo de tecnología no convencional, especie química, tipo y volumen de agua residual, concentración inicial del contaminante, pH óptimo de remoción, tiempo de contacto óptimo, temperatura, tamaño de partícula, dosis, agitación, modificación química, modelo cinético e isoterma de adsorción, capacidad máxima de adsorción y porcentaje de eficiencia.

\section{RESULTADOS}

\section{Biopolímeros empleados en la bioadsor- ción de Plomo}

De conformidad con lo expuesto, en la tabla 1 se observa que los países que han reportado más investigaciones en torno a los biopolímeros, como tecnologías no convencionales en la remoción de plomo presente en aguas residuales, corresponden a India, Malasia, China, Singapur y Nigeria; de igual forma, los reportes investigativos se han expuesto en revistas internacionales.

De acuerdo con los resultados expuestos en las tablas 1 y 2, y según la revisión bibliográfica realizada entre 2010 y 2019, correspondiente a artículos científicos relacionados con la remoción de $\mathrm{Pb}$ empleando biopolímeros, se seleccionaron nueve documentos.

Dentro de los biopolímeros que se han investigado, de esos nueve, se encuentran cinco artículos relacionados con quitosano; tres de ellos con modificación química donde la eficiencia de remoción osciló entre 95 \% a 95,3%, mientras que el quitosano sin modificación química la eficiencia fue del 80 \%,lo que indica que las modificaciones químicas mejoraron la eficiencia por encima de un 15\%.

Adicionalmente, las capacidades de máxima adsorción fueron más altas también con el quitosano modificado entre 182,5 a $189 \mathrm{mg} / \mathrm{g}$.

Por otro lado, dos artículos, de los nueve seleccionados, relacionaron a la queratina con porcentajes de remoción del 93,5\%, con una capacidad de máxima adsorción entre 32,36 y 70,42 $\mathrm{mg} / \mathrm{g}$; los valores difirieron, dado que las dosis empleadas del bioadsorbente fueron de $1 \mathrm{~g} / \mathrm{l}$ y $6 \mathrm{~g} / \mathrm{l}$, respectivamente.

Por último, los dos artículos restantes hacen referencia a dos biopolímeros como el almidón y la gelatina con polietilenimina, cuyos porcentajes de remoción corresponden al $80 \%$ y 89,9 $\%$, respectivamente, y la capacidad de máxima adsorción solo fue reportada en la gelatina modificada con un valor de $80,6 \mathrm{mg} / \mathrm{g}$. 


\section{Biorremediación empleada en la bioadsorción de plomo}

Tabla 3. Algas, levaduras, hongos y bacterias empleadas en la bioadsorción de plomo presente en aguas residuales (20102019).

\begin{tabular}{|c|c|c|c|c|c|c|}
\hline Año & Revista & País & Autor & $\begin{array}{l}\text { Tipo de bio } \\
\text { polímero }\end{array}$ & Fuente & Ref. \\
\hline 2018 & PLOS ONE & China & $\begin{array}{c}\text { Feng C.L., Li, J., Li, X., Li, K.L., Luo, K., } \\
\text { Liao, X.S. }\end{array}$ & $\begin{array}{l}\text { Verticillium insec- } \\
\text { torum } / 3\end{array}$ & $\begin{array}{l}\text { Feng et al. } \\
\text { (2018) }\end{array}$ & [14] \\
\hline 2018 & Cremosphere & China & $\begin{array}{c}\text { Wen, X., Du, C., Zeng, G., Huang, D., } \\
\text { Zhang, J., Yin, L., Tan, S., Huang, L., } \\
\text { Chen, H., Yu, G., Hu, X., Lai, C., Xu, P., } \\
\text { Wan, J. }\end{array}$ & $\begin{array}{l}\text { Bacillus licheni- } \\
\quad \text { formis }\end{array}$ & $\begin{array}{l}\text { Wen et al. } \\
\text { (2018) }\end{array}$ & [17] \\
\hline 2017 & Biotechnology Reports & India & Kalita, D. y Joshi, S. & $\begin{array}{l}\text { Pseudomonas sp. } \\
\text { W6 }\end{array}$ & $\begin{array}{c}\text { Kalita D. y } \\
\text { Joshi S. (2017) }\end{array}$ & [16] \\
\hline 2016 & Int. J. Pharm. Sci & India & Das, M. y Kumari, N. & $\begin{array}{l}\text { Enterobacter sp. y } \\
\text { Klebsiella sp. }\end{array}$ & $\begin{array}{l}\text { Das M. y Ku- } \\
\text { mari N. (2016) }\end{array}$ & [11] \\
\hline 2016 & Int. J. Environ. & India & Ojoawo, S.O., Rao, C.V. y Goveas, L.C. & Brevibacterium sp. & $\begin{array}{l}\text { Ojoawo, S.O., } \\
\text { Rao, C.V y } \\
\text { Goveas, L.C. } \\
\text { (2016) }\end{array}$ & [13] \\
\hline 2015 & $\begin{array}{l}\text { BioMed Research } \\
\text { International }\end{array}$ & España & $\begin{array}{c}\text { Muñoz, A., Espínola, F., Moya, M., Ruiz, } \\
\text { E. }\end{array}$ & Klebsiella sp. & $\begin{array}{l}\text { Muñoz et al. } \\
\text { (2015) }\end{array}$ & [10] \\
\hline 2015 & $\begin{array}{l}\text { Indian Journal of Geo- } \\
\text { Marine Sciences }\end{array}$ & India & $\begin{array}{c}\text { Kumar, D., Santhanam, S., Jayalakshmi, } \\
\text { T., Nandakumar, R.S., Ananth, A., Shen- } \\
\text { baga, D., Balaji, P. }\end{array}$ & Chlorella marina & $\begin{array}{l}\text { Kumar D. et al. } \\
\qquad(2015)\end{array}$ & [22] \\
\hline 2015 & $\begin{array}{l}\text { Water Science \& Tech- } \\
\text { nology }\end{array}$ & China & $\begin{array}{l}\text { Ye, J., Xiao, H., Xiao, B., Xu, W., Gao, L., } \\
\text { Lin, G. }\end{array}$ & $\begin{array}{l}\text { Alga roja Porphyra } \\
\text { leucosticta }\end{array}$ & Ye et al. (2015) & [18] \\
\hline 2014 & Advances in Chemistry & Egipto & $\begin{array}{c}\text { Hassan, H.H., Ali El-Shaar, Essam Khamis } \\
\text { y El-Sayed Mansour }\end{array}$ & $\begin{array}{l}\text { Enteromorpha } \\
\text { algae and its } \\
\text { silicates bonded } \\
\text { material }\end{array}$ & $\begin{array}{l}\text { Hammud et al. } \\
\qquad(2014)\end{array}$ & [21] \\
\hline 2014 & $\begin{array}{l}\text { Ciencia e Ingeniería } \\
\text { Neogranadina }\end{array}$ & Colombia & $\begin{array}{c}\text { Lissette Vizcaíno Mendoza y Natalia } \\
\text { Fuentes Molina }\end{array}$ & Algas rojas & $\begin{array}{l}\text { Vizcaino y } \\
\text { Fuentes (2014) }\end{array}$ & [12] \\
\hline 2012 & J. Environ Biol. & India & Kumar, J.I. y Oammen, C. & $\begin{array}{l}\text { Algas Spirogyra } \\
\text { hyalina }\end{array}$ & $\begin{array}{l}\text { Kumar y Oom- } \\
\text { men (2012) }\end{array}$ & [20] \\
\hline 2011 & $\begin{array}{l}\text { Journal of Medicinal } \\
\text { Plants Research }\end{array}$ & Irán & $\begin{array}{c}\text { Imani, S., Rezaei-Zarchi, S., Hashemi, } \\
\text { M., Borna, H., Javid, A., Mohamad, Z., } \\
\text { Hossein, A. }\end{array}$ & Alga Dunaliella & $\begin{array}{l}\text { Imani et al. } \\
\text { (2011) }\end{array}$ & [19] \\
\hline
\end{tabular}

Fuente: elaboración propia. 
Tabla 4. Variables óptimas de bioadsorción para plomo, empleando algas, levaduras, hongos y bacterias en aguas residuales.

\begin{tabular}{|c|c|c|c|c|c|c|c|c|c|c|c|c|c|c|}
\hline 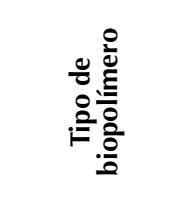 & $\begin{array}{l}\frac{0}{\pi} \\
\frac{\pi}{0} \\
\frac{\Xi}{\pi} \\
\frac{\pi}{2}\end{array}$ & 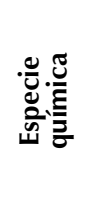 & 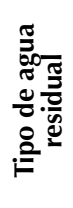 & 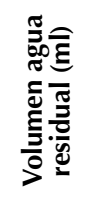 & 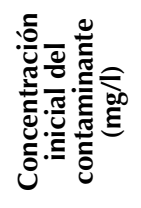 & 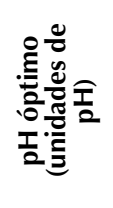 & 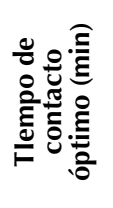 & 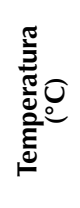 & 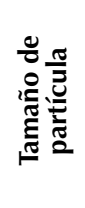 & 象 & 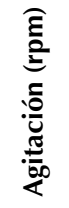 & 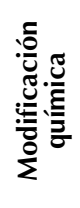 & 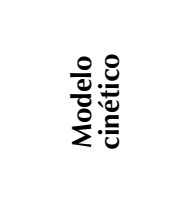 & 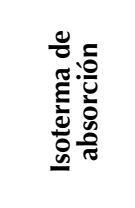 \\
\hline Klebsiella sp. & $\mathrm{Pb}$ & II & 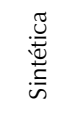 & 50 & 25 & 5 & & 30 & NA & 0,4 & 200 & NA & $\begin{array}{l}\text { Pseudosegundo } \\
\text { orden }\end{array}$ & Langmuir \\
\hline $\begin{array}{l}\text { Enterobacter sp. } \\
\text { / Klebsiella sp. }\end{array}$ & $\mathrm{Pb}$ & II & 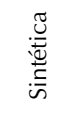 & 100 & 200 & 4 & 2880 & $\begin{array}{c}33 \mathrm{a} \\
37\end{array}$ & NA & $\mathrm{NE}$ & 180 & NA & NE & NE \\
\hline Algas rojas & $\mathrm{Pb}$ & II & 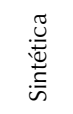 & 100 & 2,2 & 4,5 & 180 & 28 & $\mathrm{NA}$ & 50 & 200 & NA & $\mathrm{NE}$ & NE \\
\hline $\begin{array}{c}\text { Brevibacterium } \\
\text { sp. }\end{array}$ & $\mathrm{Pb}$ & II & 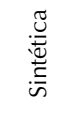 & 2000 & NE & $\begin{array}{c}6,5- \\
6,8\end{array}$ & 1440 & 37 & $\mathrm{NA}$ & $\begin{array}{c}1,0- \\
8,0\end{array}$ & 120 & NA & $\mathrm{NE}$ & $\mathrm{NE}$ \\
\hline $\begin{array}{c}\text { Verticillium } \\
\text { insectorum /3 }\end{array}$ & $\mathrm{Pb}$ & II & 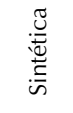 & 1000 & 250 & 4 & $\begin{array}{l}4320- \\
11520\end{array}$ & 30 & NA & 2 & 120 & NA & $\mathrm{NE}$ & $\mathrm{NE}$ \\
\hline $\begin{array}{c}\text { Pseudomonas } \\
\text { sp. W6 }\end{array}$ & $\mathrm{Pb}$ & II & 芯 & 5 & 20 & NE & 720 & 25 & $\mathrm{NA}$ & NE & 120 & NA & NE & $\mathrm{NE}$ \\
\hline $\begin{array}{c}\text { Bacillus } \\
\text { licheniformis }\end{array}$ & $\mathrm{Pb}$ & II & : & 100 & 200 & 6 & 720 & 30 & NA & 0,7 & 150 & NA & $\begin{array}{l}\text { Pseudosegundo } \\
\text { orden }\end{array}$ & Langmuir \\
\hline $\begin{array}{c}\text { Alga roja } \\
\text { Porphyra } \\
\text { leucosticta }\end{array}$ & $\mathrm{Pb}$ & II & 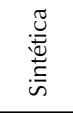 & 50 & 10 & 8 & 120 & 30 & $\mathrm{NA}$ & 15 & 150 & NA & NE & $\mathrm{NE}$ \\
\hline Alga Dunaliella & $\mathrm{Pb}$ & & 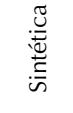 & 100 & 40 & NE & 240 & 30 & NA & NE & $\mathrm{NE}$ & NA & NE & $\mathrm{NE}$ \\
\hline $\begin{array}{c}\text { Algas Spirogyra } \\
\text { hyalina }\end{array}$ & $\mathrm{Pb}$ & II & 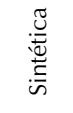 & NE & $20-80$ & NE & 120 & NE & NA & $\mathrm{NE}$ & 180 & NA & NE & Freundlinch \\
\hline $\begin{array}{c}\text { Alga } \\
\text { Enteromorpha }\end{array}$ & $\mathrm{Pb}$ & II & 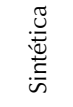 & 25 & 300 & 3 & 120 & 25 & NA & 12 & 200 & NA & $\begin{array}{c}\text { Pseudosegundo } \\
\text { orden }\end{array}$ & Langmuir \\
\hline Chlorella marina & $\mathrm{Pb}$ & II & 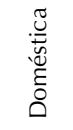 & 1000 & 0.198 & 4 & $\mathrm{NE}$ & 25 & NA & NE & NE & NA & $\mathrm{NE}$ & $\mathrm{NE}$ \\
\hline
\end{tabular}

Fuente: elaboración propia. 
Cabe resaltar que las metodologías investigativas dilucidadas en los reportes muestran su aplicación en matrices de aguas residuales sintéticas. En la tabla 3. se observa que los países que han reportado más investigaciones en torno a la biorremediación, como tecnologías no convencionales en la remoción de plomo presente en aguas residuales, son: India, China, Colombia, Corea, Egipto, España, Irán; de igual forma, los reportes investigativos se han expuesto en revistas internacionales y una nacional.

De acuerdo con los resultados de las tablas 3 y 4, de la revisión bibliográfica realizada entre 2010 y 2019, correspondiente a artículos científicos relacionados a la remoción de plomo empleando la biorremediación, se seleccionaron doce. De estos, seis hacen referencia a investigaciones con algas rojas, cinco a bacterias y los restantes a levaduras y hongos.

En cuanto a las eficiencias reportadas en algas, se encontró que estas oscilan entre el $87 \%$ y $97,3 \%$, mientras que a nivel de las bacterias el intervalo se encuentra entre el $0 \%$ al $98 \%$, donde cero se reportó con la especie Brevibacterium sp. y el $98 \%$ con la Bacillus licheniformis.

Tabla 5. Residuos agroindustriales que se han empleado en el tratamiento de aguas residuales que contienen plomo (20102019).

\begin{tabular}{|c|c|c|c|c|c|c|}
\hline Año & Revista & País & Autor & $\begin{array}{l}\text { Tipo de bio } \\
\text { polímero }\end{array}$ & Fuente & Ref. \\
\hline 2010 & $\begin{array}{l}\text { Revista de la Sociedad } \\
\text { Química del Perú }\end{array}$ & Perú & $\begin{array}{l}\text { Carmencita Lavado Meza, María del } \\
\text { Rosario Sun Kou, Salvador Bendezú }\end{array}$ & $\begin{array}{l}\text { Astillas de eu- } \\
\text { calipto (carbón } \\
\text { activado) }\end{array}$ & $\begin{array}{l}\text { Lavado, Sun } \\
\text { y Bendezú } \\
\text { (2010) }\end{array}$ & [28] \\
\hline 2010 & $\begin{array}{l}\text { Journal of Applied } \\
\text { Sciences and Environ- } \\
\text { mental Management }\end{array}$ & Irán & Asrari, E., Tavallali, H. y Hagshenas, M. & $\begin{array}{l}\text { Cascarilla de } \\
\text { arroz }\end{array}$ & $\begin{array}{c}\text { Elham, } \\
\text { Hossein y } \\
\text { Mahnoosh } \\
(2010)\end{array}$ & [33] \\
\hline 2011 & $\begin{array}{l}\text { Chemical Engineering } \\
\text { Journal }\end{array}$ & México & $\begin{array}{l}\text { J. Cruz-Olivares, C. Pérez-Alonso, C. } \\
\text { Barrera-Díaz, R. Natividad, M.C. Chapar- } \\
\text { ro-Mercado }\end{array}$ & $\begin{array}{l}\text { Pimienta de } \\
\text { Jamaica (residuo } \\
\text { obtenido de la }\end{array}$ & $\begin{array}{l}\text { Kalita D. y } \\
\text { Joshi S. (2017) }\end{array}$ & [16] \\
\hline $\begin{array}{l}\text { hidrod- } \\
\text { esti- } \\
\text { lación } \\
\text { del } \\
\text { aceite) }\end{array}$ & Cruz et al. (2011) & {$[30]$} & Das, M. y Kumari, N. & $\begin{array}{l}\text { Enterobacter sp. y } \\
\text { Klebsiella sp. }\end{array}$ & $\begin{array}{l}\text { Das M. y } \\
\text { Kumari N. } \\
(2016)\end{array}$ & [11] \\
\hline 2013 & $\begin{array}{l}\text { American Journal of } \\
\text { Analytical Chemistry }\end{array}$ & Zimbabwe & Mambo Moyo, Linda Chikazaza & $\begin{array}{c}\text { Biomasa de borla } \\
\text { de maíz }\end{array}$ & $\begin{array}{l}\text { Moyo y Chika- } \\
\text { zaza (2013) }\end{array}$ & [41] \\
\hline 2014 & $\begin{array}{l}\text { Revista de la Sociedad } \\
\text { Química del Perú }\end{array}$ & Perú & $\begin{array}{l}\text { María del Rosario Sun-Kou, Daniel } \\
\text { Obregón Valencia, Ángela Pinedo Flores, } \\
\text { Ana Lucía Paredes, Javier Aylas Orejón }\end{array}$ & Semillas de aguaje & $\begin{array}{c}\text { Sun et al } \\
(2014)\end{array}$ & [26] \\
\hline 2014 & $\begin{array}{l}\text { African Journal of Envi- } \\
\text { ronmental Science and } \\
\text { Technology }\end{array}$ & Nigeria & I.E. Agbozu y F.O. Emoruwa & Cáscara de coco & $\begin{array}{l}\text { Agbozu y } \\
\text { Emoruwa } \\
(2014)\end{array}$ & [31] \\
\hline 2014 & $\begin{array}{l}\text { American Journal of En- } \\
\text { vironmental Protection }\end{array}$ & Nigeria & $\begin{array}{l}\text { Nwankw Ogonna Daniel, Ewuim Sylva- } \\
\text { nus Chima, Mogbo Tochukwu Chinedu }\end{array}$ & Cáscara de melón & $\begin{array}{l}\text { Nwankw, Ew- } \\
\text { uim y Mogbo } \\
(2014)\end{array}$ & [37] \\
\hline
\end{tabular}

Fuente: elaboración propia. 


\begin{tabular}{|c|c|c|c|c|c|c|}
\hline 2015 & $\begin{array}{l}\text { Journal of the Taiwan } \\
\text { Institute of Chemical } \\
\text { Engineers }\end{array}$ & Serbia & $\begin{array}{c}\text { Marija Petrović, Tatjana Šoštarić, Mirjana } \\
\text { Stojanović, Jelena Milojković, Marija } \\
\text { Mihajlović, Marija Stanojević, Slavka } \\
\text { Stanković }\end{array}$ & Raw corn Silk & $\begin{array}{l}\text { Petrovíc et } \\
\text { al.(2015) }\end{array}$ & {$[23]$} \\
\hline 2015 & Prospect & Colombia & $\begin{array}{c}\text { Candelaria Tejada Tovar, Erika Ruiz } \\
\text { Paternina, Jorge Gallo Mercado, Jason } \\
\text { Moscote Bohorquez }\end{array}$ & $\begin{array}{l}\text { Bagazo de palma } \\
\text { africana }\end{array}$ & $\begin{array}{l}\text { Tejada et al. } \\
\text { (2015) }\end{array}$ & {$[24]$} \\
\hline 2015 & $\begin{array}{l}\text { Revista Acta Agronómi- } \\
\text { ca }\end{array}$ & Colombia & $\begin{array}{l}\text { Jose Herney Ramirez Franco y Maryeni } \\
\text { Karina Enríquez Enríquez }\end{array}$ & $\begin{array}{l}\text { Pseudotallo de } \\
\text { plátano (Lignina) }\end{array}$ & $\begin{array}{l}\text { Ramírez y En- } \\
\text { ríquez (2015) }\end{array}$ & {$[27]$} \\
\hline 2011 & $\begin{array}{l}\text { Journal of Medicinal } \\
\text { Plants Research }\end{array}$ & Irán & $\begin{array}{c}\text { Imani, S., Rezaei-Zarchi, S., Hashemi, } \\
\text { M., Borna, H., Javid, A., Mohamad, Z., } \\
\text { Hossein, A. }\end{array}$ & Alga Dunaliella & $\begin{array}{l}\text { Imani et al. } \\
(2011)\end{array}$ & [19] \\
\hline \multirow{3}{*}{2015} & \multirow{3}{*}{$\begin{array}{l}\text { Microporous and Meso- } \\
\text { porous Materials }\end{array}$} & \multirow{3}{*}{ Francia } & \multirow{3}{*}{$\begin{array}{c}\text { L. Largitte, T. Brudey, T. Tant, P. Couespel } \\
\text { Dumesnil, P. Lodewyck }\end{array}$} & $\begin{array}{l}\text { Semillas de } \\
\text { Guayaba (Carbón } \\
\text { activado) }\end{array}$ & \multirow{3}{*}{$\begin{array}{l}\text { Largitte et al. } \\
\text { (2015) }\end{array}$} & \multirow{3}{*}[32]{} \\
\hline & & & & $\begin{array}{l}\text { Cáscaras de } \\
\text { almendras trop- } \\
\text { icales (Carbón } \\
\text { activado) }\end{array}$ & & \\
\hline & & & & $\begin{array}{l}\text { Piedras de Dindé } \\
\text { (Carbon activado) }\end{array}$ & & \\
\hline 2015 & $\begin{array}{l}\text { Journal of Industrial and } \\
\text { Engineering Chemistry }\end{array}$ & Zimbabwe & $\begin{array}{c}\text { Mambo Moyo, Upenyu Guyo, Grace } \\
\text { Mawenyiyo, Ngceboyakwethu P.Zin- } \\
\text { yama, Benias C.Nyamunda }\end{array}$ & $\begin{array}{l}\text { Cáscara de } \\
\text { semilla de marula } \\
\text { (Sclerocarya } \\
\text { birrea) }\end{array}$ & $\begin{array}{l}\text { Moyo et al. } \\
\quad(2015)\end{array}$ & [35] \\
\hline 2015 & $\begin{array}{l}\text { World Academy of Sci- } \\
\text { ence, Engineering and } \\
\text { Technology Internation- } \\
\text { al Journal of Environ- } \\
\text { mental and Ecological } \\
\text { Engineering }\end{array}$ & Nigeria & $\begin{array}{l}\text { W. E. Igwegbe, B.C. Okoro y J.C. Osuag- } \\
\text { wu }\end{array}$ & $\begin{array}{l}\text { Tronco leñoso de } \\
\text { papaya (Carica } \\
\text { papaya) }\end{array}$ & $\begin{array}{l}\text { Igwegbe, Oko- } \\
\text { ro y Osuagwu } \\
(2015)\end{array}$ & {$[40]$} \\
\hline 2017 & New Biotechnology & Romania & $\begin{array}{c}\text { Irina Morosanu, Carmen Teodosiu, } \\
\text { Carmen Paduraru, Dumitrita Ibanescu, } \\
\text { Lavinia Tofan }\end{array}$ & $\begin{array}{l}\text { Biomasa de colza } \\
\text { (Brassica napus L.) }\end{array}$ & $\begin{array}{l}\text { Morosanu et } \\
\text { al. (2017) }\end{array}$ & [36] \\
\hline \multirow{2}{*}{2018} & \multirow{2}{*}{$\begin{array}{l}\text { Biotecnología en el } \\
\text { Sector Agropecuario y } \\
\text { Agroindustrial }\end{array}$} & \multirow{2}{*}{ Colombia } & \multirow{2}{*}{$\begin{array}{c}\text { Candelaria Tejada Tovar, Jorge Gallo Mer- } \\
\text { cado, Jeison Moscote, Angel Villabona, } \\
\text { Diofanor Acevedo Correa }\end{array}$} & $\begin{array}{l}\text { Bagazo de palma } \\
\text { africana }\end{array}$ & \multirow{2}{*}{$\begin{array}{l}\text { Tejada et al. } \\
\text { (2018) }\end{array}$} & \multirow{2}{*}{ [25] } \\
\hline & & & & Cáscaras de ñame & & \\
\hline 2018 & Applied Water Science & India & $\begin{array}{c}\text { Poonam, Sushi Kumar Bharti y Narendra } \\
\text { Kumar }\end{array}$ & $\begin{array}{l}\text { Bagazo de la } \\
\text { caña de azúcar } \\
\text { (Biochar) }\end{array}$ & $\begin{array}{l}\text { Poonam, Ku- } \\
\text { mar y Kumar } \\
\quad(2018)\end{array}$ & [29] \\
\hline \multirow[b]{2}{*}{2018} & \multirow{2}{*}{$\begin{array}{l}\text { Journal of Environmen- } \\
\text { tal Chemical Engineer- } \\
\text { ing }\end{array}$} & \multirow[b]{2}{*}{ Indonesia } & \multirow{2}{*}{$\begin{array}{l}\text { Eddy Heraldy, Witri Wahyu Lestari, Diah } \\
\text { Permatasari, Devita Dwi Arimurti }\end{array}$} & $\begin{array}{l}\text { Residuos del jugo } \\
\text { de manzana }\end{array}$ & \multirow{2}{*}{$\begin{array}{l}\text { Heraldy et al. } \\
\quad(2018)\end{array}$} & \multirow[b]{2}{*}[34]{} \\
\hline & & & & $\begin{array}{l}\text { Residuos de } \\
\text { tomate }\end{array}$ & & \\
\hline 2018 & $\begin{array}{l}\text { International Journal of } \\
\text { Engineering and Tech- } \\
\text { nology (IJET) }\end{array}$ & Colombia & $\begin{array}{l}\text { Candelaria Tejada Tovar, Angel Villabona } \\
\text { Ortiz, Diofanor Acevedo Correa, Norida } \\
\text { Pajaro Gómez, María Otero Amor }\end{array}$ & $\begin{array}{l}\text { Cáscara de limón } \\
\text { (Citrus limonum) }\end{array}$ & $\begin{array}{l}\text { Tejada et al. } \\
\text { (2018) }\end{array}$ & [38] \\
\hline 2019 & $\begin{array}{l}\text { Chemical Engineering } \\
\text { Transactions }\end{array}$ & Malasia & $\begin{array}{c}\text { Abdul Rahman Abdul Rahim, Nurul Ekmi } \\
\text { Rabat, Khairiraihanna Johari, Norasikin } \\
\text { Saman y Hanapi Mat }\end{array}$ & $\begin{array}{l}\text { Residuos de coco } \\
\text { desecados }\end{array}$ & $\begin{array}{l}\text { Abdul et al. } \\
\quad(2019)\end{array}$ & [39] \\
\hline
\end{tabular}


Tabla 6. Variables óptimas del proceso de remoción de plomo, empleando residuos agroindustriales

\begin{tabular}{|c|c|c|c|c|c|c|c|c|c|c|c|c|c|c|c|c|c|c|}
\hline 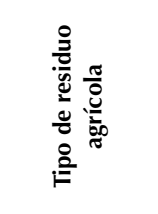 & $\frac{\frac{8}{\pi}}{\frac{d}{2}}$ & 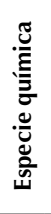 & 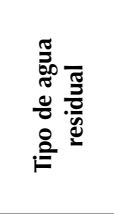 & 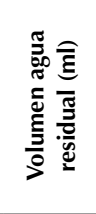 & 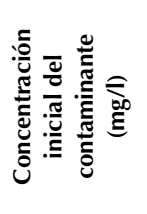 & 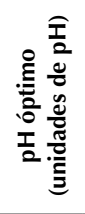 & 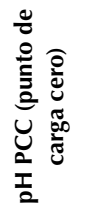 & 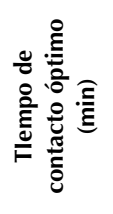 & 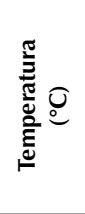 & 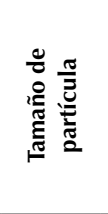 & 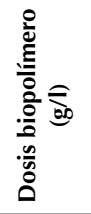 & 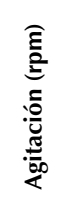 & 冚 & 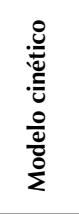 & 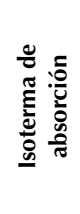 & 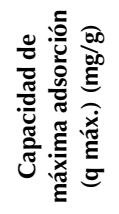 & 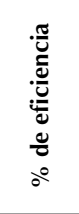 & 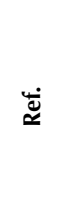 \\
\hline Raw corn silk & $\mathrm{Pb}$ & II & 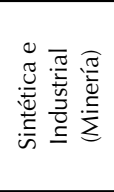 & 250 & 200 & 200 & 6.0 & 120 & 25 & $\begin{array}{l}1.357 \\
\mathrm{~m} 2 / \mathrm{g}\end{array}$ & 1.0 & 250 & $\stackrel{\Sigma}{z}$ & 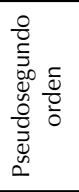 & 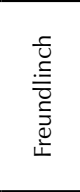 & 90 & 88,5 & [23] \\
\hline $\begin{array}{c}\text { Bagazo } \\
\text { de palma } \\
\text { africana }\end{array}$ & $\mathrm{Pb}$ & II & 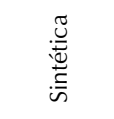 & NE & 100 & 100 & $\mathrm{~N} / \mathrm{A}$ & 10 & 25 & $1 \mathrm{~mm}$ & 5.0 & 150 & 道总 & $\begin{array}{l}\frac{\bar{v}}{\partial} \\
\frac{o}{W}\end{array}$ & 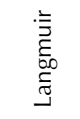 & 461 & 99.56 & [24] \\
\hline $\begin{array}{c}\text { Bagazo } \\
\text { de palma } \\
\text { africana }\end{array}$ & $\mathrm{Pb}$ & II & \multirow{2}{*}{ 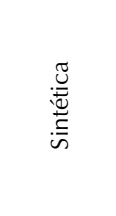 } & \multirow{2}{*}{500} & \multirow{2}{*}{100} & \multirow[t]{2}{*}{100} & \multirow[t]{2}{*}{$\mathrm{N} / \mathrm{A}$} & \multirow[t]{2}{*}{150} & \multirow{2}{*}{25} & \multirow{2}{*}{$0.5 \mathrm{~mm}$} & \multirow{2}{*}{20.0} & \multirow{2}{*}{200} & \multirow{2}{*}{ 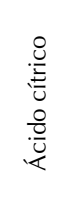 } & \multirow{2}{*}{$\begin{array}{l}\tilde{\Xi} \\
\stackrel{\Xi}{E} \\
\stackrel{F}{F}\end{array}$} & \multirow[t]{2}{*}{ 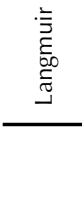 } & 62.63 & 98.04 & [25] \\
\hline $\begin{array}{l}\text { Cáscara de } \\
\text { ñame }\end{array}$ & $\mathrm{Pb}$ & II & & & & & & & & & & & & & & 44.56 & \multicolumn{2}{|l|}{99.20} \\
\hline $\begin{array}{c}\text { Semillas de } \\
\text { aguaje }\end{array}$ & $\mathrm{Pb}$ & II & 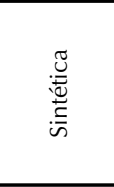 & 20 & $2-80$ & $2-80$ & 2.55 & 240 & 25 & $2.6 \mathrm{~nm}$ & & & $\begin{array}{l} \pm \\
0 \\
\frac{1}{M} \\
\text { I }\end{array}$ & 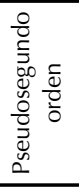 & 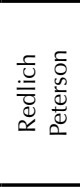 & 74.80 & $\mathrm{NE}$ & [26] \\
\hline $\begin{array}{c}\text { Pseudotallo } \\
\text { de plátano } \\
\text { (lignina) }\end{array}$ & $\mathrm{Pb}$ & II & 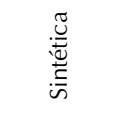 & $\mathrm{NE}$ & $124-207$ & $\begin{array}{l}124- \\
207\end{array}$ & $\mathrm{~N} / \mathrm{A}$ & 30 & 25 & $\begin{array}{c}0.30 \\
\mathrm{~cm} 3 / \mathrm{g}\end{array}$ & 2.5 & $\mathrm{NE}$ & $\begin{array}{l} \pm \\
\text { N } \\
\text { I }\end{array}$ & $\mathrm{Z}$ & 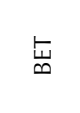 & 23.27 & 55.25 & [27] \\
\hline $\begin{array}{c}\text { Astillas de } \\
\text { eucalipto } \\
\text { (Carbón } \\
\text { activado) }\end{array}$ & $\mathrm{Pb}$ & II & 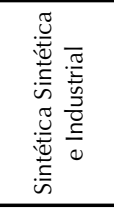 & 50 & $50-500$ & $\begin{array}{l}50- \\
500\end{array}$ & 3.0 & 180 & 25 & $\begin{array}{l}761.1 \\
\mathrm{~m} 2 / \mathrm{g}\end{array}$ & 1.0 & 500 & $\begin{array}{l} \pm \\
0 \\
\frac{0}{1} \\
M\end{array}$ & 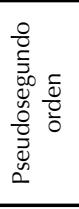 & 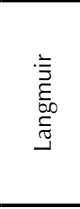 & 142.10 & 99.70 & [28] \\
\hline $\begin{array}{c}\text { Bagazo } \\
\text { de la caña } \\
\text { de azúcar } \\
\text { (Biochar) }\end{array}$ & $\mathrm{Pb}$ & II & 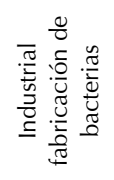 & 10000 & $\begin{array}{c}2.393 \pm \\
0.030\end{array}$ & $\begin{array}{c}2.393 \\
\pm \\
0.030\end{array}$ & $\mathrm{~N} / \mathrm{A}$ & 140 & $25 \pm 3$ & $\begin{array}{c}12.628 \\
\pm 0.30 \\
\mathrm{~m} 2 / \mathrm{g}\end{array}$ & 5.0 & 120 & 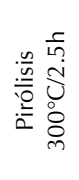 & 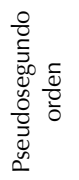 & 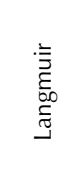 & 12.741 & 75.38 & [29] \\
\hline $\begin{array}{c}\text { Pimienta } \\
\text { de Jamaica } \\
\text { (Pimenta } \\
\text { dioica L. } \\
\text { Merril) }\end{array}$ & $\mathrm{Pb}$ & & 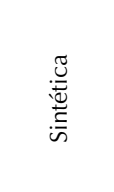 & $N E$ & 25 & 25 & N/A & 90 & 35 & $\begin{array}{c}0.836 \\
\mathrm{~mm}\end{array}$ & 1.0 & 200 & $\$$ & 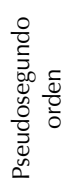 & 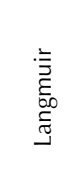 & 22.37 & 95.50 & [30] \\
\hline $\begin{array}{l}\text { Cáscara de } \\
\text { coco }\end{array}$ & $\mathrm{Pb}$ & II & 莺 & 50 & 0.09 & 0.09 & N/A & 60 & 25 & $\begin{array}{l}120 \\
\mathrm{~mm}\end{array}$ & 24.0 & $\mathrm{NE}$ & $\stackrel{s}{z}$ & 㞱 & 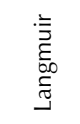 & 0.00039 & 81.10 & [31] \\
\hline $\begin{array}{l}\text { Semillas de } \\
\text { guayaba } \\
\text { (Psidium } \\
\text { Guajava) }\end{array}$ & $\mathrm{Pb}$ & II & $\widetilde{\widetilde{U}}$ & & & & 9.60 & & & 1000 & & & $\begin{array}{l}\text { ज̃ } \\
\text { ड़ } \\
8 \\
0\end{array}$ & $\begin{array}{l}\frac{c}{0} \\
\frac{0}{0} \\
\end{array}$ & 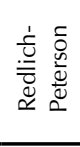 & 96 & & \\
\hline $\begin{array}{c}\text { Cáscaras de } \\
\text { almendras } \\
\text { tropicales } \\
\text { (Acrocomia } \\
\text { karukerana) }\end{array}$ & $\mathrm{Pb}$ & II & : & 1000 & 90 & 90 & 10.30 & 6000 & 30 & $\mathrm{~m} 2 / \mathrm{g}$ & 0.5 & $\mathrm{NE}$ & 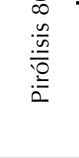 & 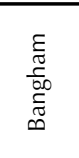 & $\begin{array}{l}\text { స్ } \\
\frac{\pi}{2}\end{array}$ & 112 & 100 & [32] \\
\hline
\end{tabular}


Fuente: elaboración propia.

\begin{tabular}{|c|c|c|c|c|c|c|c|c|c|c|c|c|c|c|c|c|c|c|}
\hline 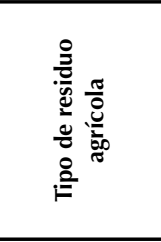 & 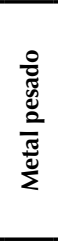 & 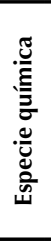 & 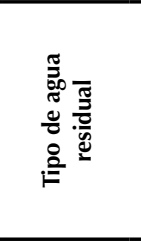 & 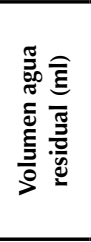 & 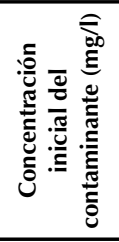 & 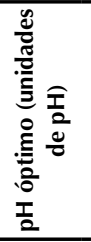 & 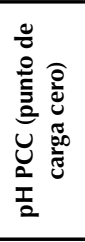 & 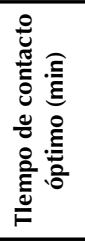 & 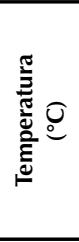 & 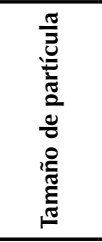 & 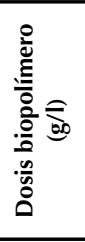 & 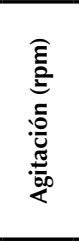 & 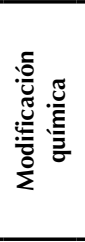 & 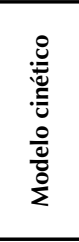 & 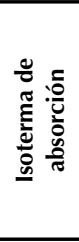 & 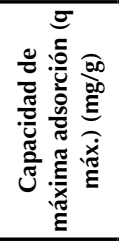 & 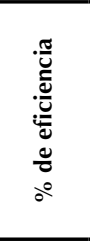 & $\dot{\ddot{\varpi}}$ \\
\hline $\begin{array}{l}\text { Piedras } \\
\text { de Dindé } \\
\text { (Terminalia } \\
\text { Catappa) }\end{array}$ & $\mathrm{Pb}$ & & & & & & 6.80 & & & & & & & & 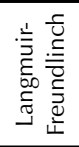 & ำ & & [33] \\
\hline $\begin{array}{c}\text { Cascarilla de } \\
\text { arroz }\end{array}$ & $\mathrm{Pb}$ & II & 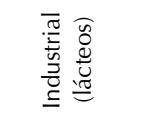 & 30 & 0.05 & 9.0 & $\mathrm{~N} / \mathrm{A}$ & 60 & $25 \pm 1$ & $\mathrm{NE}$ & 33.0 & NE & $\stackrel{\S}{Z}$ & 㞱 & 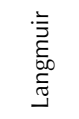 & 0.6216 & 96.80 & [34] \\
\hline $\begin{array}{c}\text { Residuos } \\
\text { del jugo de } \\
\text { manzana }\end{array}$ & $\mathrm{Pb}$ & II & 节 & 25 & ח 100 & 40 & N/A & 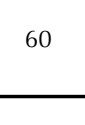 & 25 & 100 & 40 & 120 & I & $\begin{array}{l}\frac{c}{0} \\
\frac{0}{0} \\
\frac{0}{0} \\
\vdots\end{array}$ & 言 & 108 & 0400 & {$[25]$} \\
\hline $\begin{array}{l}\text { Residuos del } \\
\text { tomate }\end{array}$ & $\mathrm{Pb}$ & & & & & & & 90 & & & & & & $\begin{array}{l}\frac{\breve{o}}{0} \\
\frac{0}{3} \\
w \\
0\end{array}$ & & 152 & & \\
\hline $\begin{array}{c}\text { Cáscara } \\
\text { de semilla } \\
\text { de marula } \\
\text { (Sclerocarya } \\
\text { birrea) }\end{array}$ & $\mathrm{Pb}$ & II & 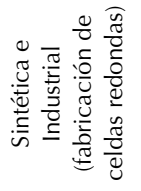 & 50 & 1000 & 5.0 & N/A & 60 & 20 & $150 \mu \mathrm{m}$ & 10.0 & 4000 & 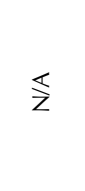 & 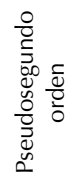 & 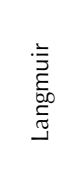 & 20 & 97.40 & [36] \\
\hline $\begin{array}{l}\text { Biomasa } \\
\text { de colza } \\
\text { (Brassica } \\
\text { napus L.) }\end{array}$ & $\mathrm{Pb}$ & II & 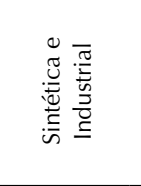 & 1000 & 1000 & 5.2 & 4.0 & 180 & $\mathrm{NE}$ & $\begin{array}{c}0.1-0.2 \\
\mathrm{~mm}\end{array}$ & 12.0 & NE & $\$$ & 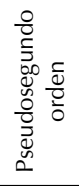 & 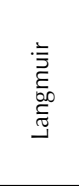 & 21.29 & 94.47 & [37] \\
\hline $\begin{array}{l}\text { Cáscara } \\
\text { de melón } \\
\text { (Citrullus } \\
\text { colocynthis) }\end{array}$ & $\mathrm{Pb}$ & II & 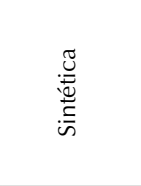 & 1000 & 1000 & $\mathrm{NE}$ & N/A & 10 & 25 & $\begin{array}{l}250- \\
500 \\
\mu \mathrm{m}\end{array}$ & 0.5 & 150 & 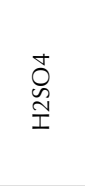 & 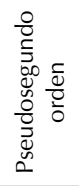 & 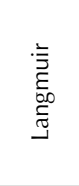 & $\mathrm{NE}$ & 100 & [38] \\
\hline $\begin{array}{l}\text { Cáscara de } \\
\text { limón (Citrus } \\
\text { limonum) }\end{array}$ & $\mathrm{Pb}$ & II & 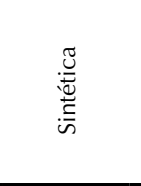 & 1000 & 100 & 6.0 & N/A & 310 & & $\begin{array}{c}0.500 \\
\mathrm{~mm}\end{array}$ & & 200 & 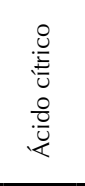 & 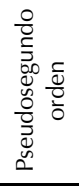 & 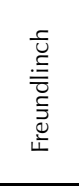 & $\begin{array}{c}19.56 \\
1.21\end{array}$ & $\begin{array}{l}97.78 \\
93.83\end{array}$ & \\
\hline $\begin{array}{l}\text { Residuos } \\
\text { de coco } \\
\text { desecados }\end{array}$ & $\mathrm{Pb}$ & II & 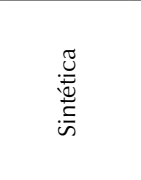 & 25 & 50 & 6.0 & $\mathrm{~N} / \mathrm{A}$ & 1440 & 30 & $\begin{array}{c}150 \\
-300 \\
\mu \mathrm{m}\end{array}$ & 1.0 & 200 & $\$$ & 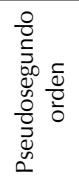 & 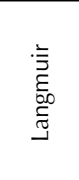 & 55.86 & $\mathrm{NE}$ & [39] \\
\hline $\begin{array}{c}\text { Tronco leñoso } \\
\text { de papaya } \\
\text { (Carica } \\
\text { papaya } \\
\text { Linnaeus) } \\
\end{array}$ & $\mathrm{Pb}$ & II & 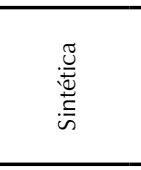 & 100 & 50 & 5.9 & N/A & 180 & 25 & $\begin{array}{l}2.50 \\
\mathrm{~mm}\end{array}$ & 10.0 & 160 & $\stackrel{\zeta}{z}$ & $\stackrel{\mathrm{z}}{\mathrm{z}}$ & $\stackrel{\amalg}{Z}$ & $\mathrm{NE}$ & $\mathrm{NE}$ & [40] \\
\hline $\begin{array}{l}\text { Biomasa de } \\
\text { borla de maíz }\end{array}$ & $\mathrm{Pb}$ & II & 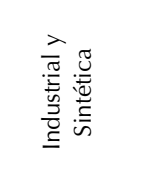 & 100 & 10 & 5.4 & $\mathrm{~N} / \mathrm{A}$ & 60 & $25 \pm 1$ & $\begin{array}{c}100 \\
-300 \\
\mu \mathrm{m}\end{array}$ & 1.2 & & $\begin{array}{l}\text { O } \\
\stackrel{N}{I} \\
\text { I }\end{array}$ & 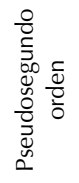 & 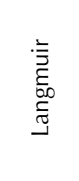 & 37.31 & 93.9 & [41] \\
\hline
\end{tabular}


Por último, con relación al parámetro de capacidad de máxima absorción el que reportó un mayor valor fue con la bacteria Klebsiella sp con 140,19 mg*g-1. Cabe resaltar que las metodologías investigativas dilucidadas en los reportes investigativos muestran su aplicación en matrices de aguas residuales sintéticas.

En la tabla 5, se observa que los países que han reportado más investigaciones en torno a los residuos agroindustriales, como tecnologías no convencionales en la remoción de plomo presente en aguas residuales, corresponden a Colombia, Nigeria, Perú, India, Indonesia, Francia, Irán, Malasia, México, Romania, Serbia y Zimbabue. De igual forma, los reportes investigativos se han expuesto en revistas internacionales y nacionales, y que este tipo de tecnologías son las de más tendencia en investigación según la indagación realizada, donde son 24 residuos agroindustriales los que se han investigado, y que su aplicación se ha dado tanto en aguas residuales sintéticas y industriales (para algunos).

Con relación a las tablas 5 y 6 , ha sido amplio cada uno de los residuos agrícolas empleados para la remoción de plomo, entre los cuales se encuentran desde semillas, cáscaras de diferentes frutas, bagazo, pseudotallo de plátano, astillas de un material vegetal, residuos de diferentes jugos, entre otros. A su vez, con respecto a las variables óptimas de adsorción se presenta que los residuos agrícolas de mayor eficiencia con un $100 \%$ se reportan las semillas de guayaba y cáscara de melón y alrededor del $98 \%$ al $99 \%$ se encuentran las astillas de eucalipto, la cáscara de ñame y el bagazo de palma africana.

\section{CONCLUSIONES}

1. Al realizar un comparativo entre los tres tipos de tecnologías no convencionales (biopolímeros, biorremediación y residuos agroindustriales) se observa que las publicaciones que más se han reportado son las de los residuos agroindustriales con respecto a las demás. A su vez, es importante indicar que los porcentajes de mayor eficiencia con un $100 \%$ corresponden a las semillas de guayaba y la cáscara de melón, seguidas de las técnicas de biorremediación con porcentajes del $98 \%$ y la de biopolímeros entre el $80 \%$ al $95 \%$.

2. Es importante indicar que, para obtener los porcentajes altos de remoción para los metales pesados, es necesario optimizar cada una de las variables en las técnicas no convencionales como el $\mathrm{pH}$, la temperatura, el tamaño de partícula, el tiempo de contacto, la cantidad de biomasa, el tiempo de agitación y la concentración inicial de la solución del contaminante.

3. Las cinéticas de adsorción que se reportaron en las tres técnicas no convencionales se encuentran las de pseudosegundo orden, Elovich, Thomas y Bangham.

4. Las isotermas de adsorción que se reportaron en las tres técnicas no convencionales se encuentran las de Langmuir, Freundlich, Redlich-Peterson, Themkin y Khan.

5. Al llevar a cabo este tipo de revisiones, se pretende que las industrias que viertan plomo en sus aguas resid- 
uales apliquen este tipo de tecnologías, por sus altos porcentajes de remoción, de fácil adquisición, económicos y, lo más importante, cumplan las normas ambientales y que no generen más impactos negativos al ecosistema.

\section{FINANCIAMIENTO}

La presente investigación no recibió financiamiento.

\section{REFERENCIAS}

Abadin, H., Ashizawa, A., Stevens, Y.W. et al. (2007). Toxicological profile for lead. Atlanta, GA: Agency for Toxic Substances and Disease Registry, Public Health Statement. Recuperado de https://www.ncbi.nlm.nih.gov/books/ NBK158768/

Abdul Rahim A.R., Rabat N.E., Johari K., Saman N. y Mat H. (2019). Removal of lead (ii) ions from aqueous solution using desiccated coconut waste as lowcost adsorbent. Chemical Engineering Transactions, 72, 169-174 DOI: 10.3303/CET1972029

Ablouh, E., Hanani, Z., Eladlani, N. et al. (2019). Chitosan microspheres/sodium alginate hybrid beads: an efficient green adsorbent for heavy metals removal from aqueous solutions. Sustainable Environment Research, 29. DOI: https://doi.org/10.1186/s42834-0190004-9

Agbozu, I.E. y Emoruwa, F. (2014). Batcha adsorption on hevay metals $(\mathrm{Cu}, \mathrm{Pb}$, $\mathrm{Fe}, \mathrm{Cr}$ and $\mathrm{Cd}$ ) from aqueous solutions using coconut husk. African Journal of Enviromental Sciencie and Technology, 8(4). https://doi.org/10.5897/ AJEST2013.1577

Agency for Toxic Substances and Disease Registry (ATSDR) (2007). Toxicological profile for lead (Update). Atlanta, GA: Department of Health and Human Services, Public Health Service.

Anantha, R. K., \& Kota, S. (2016). Removal of lead by adsorption with the renewable biopolymer composite of feather ( Dromaius novaehollandiae ) and chitosan ( Agaricus bisporus ). Environmental Technology \& Innovation, 6, 11-26. doi: https://doi.org/10.1016/j. eti.2016.04.004

Amaringo, F. y Hormaza A. (2018). Adsorción de rojo 40 sobre cascarilla de arroz: determinación del equilibrio, cinética y termodinámica. Revista Tecnura, 22(56), 13-28. DOI: https://doi. org/10.14483/22487638.12961

Asrari, E., Tavallali, H. y Hagshenas, M. (2010). Removal of Zn (II) and Pb (II) ions Using Rice Husk in Food Industrial Wastewater. Journal of Applied Sciences and Environmental Management, 14(4), 159-162. https://www. ajol.info/index.php/jasem/article/viewFile/63306/51190 DOI: https://doi. org/10.4314/jasem.v14i4.63306

Baharuddin, N.H, Meriam, N., Sulaiman, N., Aroua, M.K., Ghazali, M., Nawawi, M., Kassim, M.A., Othman, M.R. e Dahlan, I. (2019). Starch as novel water soluble biopolymer in removal mixtures heavy metal ions via polymer enhanced ultrafiltration. AIP Conference Proceedings 2124, 030012. DOI: https://doi.org/10.1063/1.5117134 
Bamgbose, J.T., Adewuyi, S., Bamgbose O. y Adetoye A.A. (2010). Adsorption kinetics of cadmium and lead by chitosan. African Journal of Biotechnology, 9(17), 2560-2565.

Barthelmy, D. (2014). Webmineral database. Recuperado de: http://www. webmineral.com/chem/Chem-Pb. shtml\#.Xi3T0U9KgY0

Castañeda, M., Mirasol, M., Raymundo, L. y Solidum, J. (2012). Biosorption and Desorption of Lead $(\mathrm{Pb}+2)$ from Simulated WasteWater Using Freshwater Snail Shells, Melanoides tuberculata Muller (Family Thiaridae). 2nd International Conference on Environment and BioScience IPCBEE, (44). DOI: https://doi.org/10.7763/ipcbee

Caviedes Rubio, D.I., Muñoz Calderón, R.A., Perdomo Gualtero, A., Rodríguez Acosta, D. y Sandoval Rojas, I.J. (2015). Tratamientos para la remoción de metales pesados comúnmente presentes en aguas residuales industriales. Una Revisión. Revista de Ingeniería y Región, 13(1), 73-90. https:// doi.org/10.25054/22161325.710

Consejería de Presidencia y Portavoz del Gobierno Comunidad de Madrid (2005). Ley 10/1993, sobre vertidos líquidos industriales al sistema integral de saneamiento. Madrid. Recuperado de: http://www.madrid.org/wleg_pub/ secure/normativas/contenidoNormativa.jsf?opcion=VerHtml\&nmnorma $=374 \&$ cdestado=P\#no-back-button

Cruz-Olivares, J., Pérez-Alonso, C., Barrera-Díaz, C., Natividad, R. y Chaparro-Mercado, M.C. (2011). Thermo- dynamical and analytical evidence of lead ions chemisorption onto Pimenta dioica. Chemical Engineering Journal, 166(3), 814-821. DOI: https:// doi.org/10.1016/j.cej.2010.11.041

Das, M. y Kumari, N. (2016). A microbial bioremediation approach: Removal of heavy metal using isolated bacterial strains from industrial effluent disposal site. International Journal of Pharmaceutical Sciences Review and Research, 38(1), 111-114. Recuperado de https://pdfs.semanticscholar.org/c4ad/b17bd924bb31d185f86a6883a4cff2fdd0f7.pdf

Feng, C., Li, J., Li, X., Li, K., Luo, K. y Liao X.S. et al. (2018). Characterization and mechanism of lead and zinc biosorption by growing Verticillium insectorum J3. PLoS ONE, 13(12), e0203859. DOI: https://doi. org/10.1371/journal.pone.0203859

García Vaca, M. C., García Ubaque, C. A., y de Plaza Solórzano, J. S. (2016). Estudio exploratorio del tratamiento de agua de lavado de tintas por método de electrocoagulación/electroflotación. Revista Tecnura, 20(47), 107-117. https://doi.org/10.14483/ udistrital.jour.tecnura.2016.1.a09

Gómez, D. L., Rodríguez, J. P., Esteban, J. A., y Betancur P., J. F.. (2019). Coffee Pulp: A Sustainable Alternative Removal of $\mathrm{Cr}(\mathrm{VI})$ in Wastewaters. Processes, 7(403), 1-12. Doi: https://doi. org/10.3390/pr7070403

Hammud, H.H., El-Shaar, A., Khamis, E. y Mansour, E.S. (2014) Estudios de adsorción de plomo por algas deromante y sus materiales unidos de silicatos. 
Avances en Química, 2014,1-11. DOI: https://doi.org/10.1155/2014/205459

Heraldy, E., Lestari, W.W., Permatasari, D. y Arimurti, D.D. (2018). Biosorbent from tomato waste and apple juice residue for lead removal. Journal of Environmental Chemical Engineering, 6(1), 1201-1208. DOI: https://doi. org/10.1016/j.jece.2017.12.026

Igwegbe, W.E., Okoro, B.C. y Osuagwu, J.C. (2015). Use of Carica papaya as a bio-sorbent for removal of heavy metals in wastewater. World Academy of Science, Engineering and Technology International Journal of Environmental and Ecological Engineering 9(12), 14001404.

Imani, S., Rezaei-Zarchi, S., Hashemi, M., Borna, H., Javid, A., Mohamad, Z. y Hossein, B.A. (2011). $\mathrm{Hg}, \mathrm{Cd}$ and $\mathrm{Pb}$ heavy metal bioremediation by Dunaliella alga. Journal of Medicinal Plants Research, 5(13), 2775-2780.

Kalita, D. y Joshi, S.R. (2017). Estudio sobre biorremediación de plomo por exopolisacárido productor de bacteria metalofílica aislada de hábitat extremo. Biotechnology Reports, 16, 48-57. DOI: https:// doi.org/10.1016/j.btre.2017.11.003

Kong, J., Yue, Q., Sun, S., Gao, B., Kan, Y., Li, Q. y Wang, Y. (2014). Adsorption of Pb(II) from aqueous solution using keratin waste - hide waste: Equilibrium, kinetic and thermodynamic modeling studies. Chemical Engineering Journal, 241, 393-400. Doi: https://doi.org/10.1016/j. cej.2013.10.070

Kumar Dinesh, S.P., Santhanam, T., Jayalakshmi, R., Nandakumar, S., Ananth, A.,
Shenbaga, D. y Prasath, B. (2015). Excessive nutrients and heavy metals removal from diverse waste waters using marine microalga Chlorella marina (Butcher). Indian Journal of Geo-Marine Sciences, 44(1), 97-103X-XX.

Largitte, L., Brudey, T., Tant, T., Dumesnil, P.C. y Lodewyckx, P. (2016). Comparación de la adsorción de plomo por carbonos activados de tres precursores lignocelulósicos. Materiales Microporosos y Mesoporosos, 219, 265-275. DOI: $\quad$ https://doi.org/10.1016/j.micromeso.2015.07.005

Lavado Meza, C., Sun Kou, M. y Bendezú, S. (2010). Adsorción de plomo de efluentes industriales usando carbones activados con H3PO4. Revista de la Sociedad Química del Perú, 76(2),165178.

Legislación Secundaria del Ministerio de Ambiente de Guayaquil (2004). Norma de calidad ambiental y de descarga de efluentes: recurso agua. Guayaquil. Recuperado de: http://www.industrias.ec/ archivos/CIG/file/CARTELERA/ReformaAnexo28feb2014FINAL.pdf

León A., A., Córdoba R., J.C. y Carreño S., U.F. (2016). Revisión del estado de arte en captación y aprovechamiento de aguas lluvias en zonas urbanas y aeropuertos. Revista Tecnura, 20(50), 141-153. DOI: 10.14483/udistrital.jour.tecnura.2016.4.a10

Li, B., Zhou, F., Huang, K., Wang, Y., Mei, S., Zhou, Y. y Jing, T. (2016). Highly efficient removal of lead and cadmium during wastewater irrigation using a polyethylenimine-grafted gelatin 
sponge. Scientific Reports, 6(1). DOI: https://doi.org/10.1038/srep33573

Londoño Franco, L.F., Londoño Muñoz, P.T. y Muñoz García, F.G. (2016). Los riesgos de los metales pesados en la salud humana y animal. Biotecnología en el Sector Agropecuario y Agroindustrial, 14(2), 145-153. DOI: https://doi. org/10.18684/BSAA(14)145-153

Manzoor, K., Ahmad, M., Ahmad, S. y Ikram, S. (2019). Removal of Pb(ii) and Cd(ii) from wastewater using arginine cross-linked chitosan-carboxymethyl cellulose beads as green adsorbent. RSC Advances, 9(14), 7890-7902. DOI: https://doi.org/10.1039/C9RA00356H

Ministerio de Ambiente y Recursos Naturales de Guatemala (2005). Reglamento de descargas de aguas residuales a cuerpos receptores: Acuerdo Gubernativo No. 66-2005. Guatemala.

Morosanu, I., Teodosiu, C., Paduraru, C., lbanescu, D. y Tofan, L. (2017). Biosorption of lead ions from aqueous effluents by rapeseed biomass. New Biotechnology, 39, 110-124. DOI: https://doi. org/10.1016/j.nbt.2016.08.002

Moyo, M. y Chikazaza, L. (2013). Bioremediation of Lead(II) from Polluted Wastewaters Employing Sulphuric Acid Treated Maize Tassel Biomass. American Journal of Analytical Chemistry, 4(12), 689-695. DOI: https://doi.org/10.4236/ ajac.2013.412083

Moyo, M., Guyo, U., Mawenyiyo, G., Zinyama, N.P. y Nyamunda, B.C. (2015). Marula seed husk (Sclerocarya birrea) biomass as a low cost biosorbent for removal of $\mathrm{Pb}(\mathrm{II})$ and $\mathrm{Cu}(\mathrm{II})$ from aqueous solution. Journal of Industrial and Engineering Chemistry, 27, 126132. DOI: https://doi.org/10.1016/j. jiec.2014.12.026

Muñoz, A.J., Espínola, F., Moya, M. y Ruiz, E. (2015). Biosorption of $\mathrm{Pb}(\mathrm{II})$ Ions byKlebsiellasp. 3S1 Isolated from a Wastewater Treatment Plant: Kinetics and Mechanisms Studies. BioMed Research International, 2015, 1-12. DOI: https://doi.org/10.1155/2015/719060

(II) Ions byKlebsiellasp. 3S1 Isolated from a Wastewater Treatment Plant: Kinetics and Mechanisms Studies. BioMed Research International, 2015, 1-12. DOI: 10.1155/2015/719060 https://doi. org/10.1155/2015/719060

Ngah, W.S.W. y Fatinathan, S. (2010). Pb(II) biosorption using chitosan and chitosan derivatives beads: Equilibrium, ion exchange and mechanism studies. Journal of Environmental Sciences, 22(3), 338-346. DOI: https://doi.org/10.1016/ S1001-0742(09)60113-3

Nwankwo, D., Sylvanus Chima, E. y Tochukwu, M. (2014). Comparative study of the Bioadsorbtion of Cadmium and lead from industrial waste water using melon (citrullus colocynthis) husk activated with sulphuric acid. American Journal of Environmental Protection, 1(1), 1-8. DOI: 10.11648/j. ajep.s.20140101.11.

Ojeda Suárez, R., Spoor, M. y Estrada, M.E. (2017). El índice desempeño ambiental y la resiliencia social en los ecosistemas. Universidad y Sociedad, 9(1), 6-12. Recuperado de http://rus. ucf.edu.cu/ 
Ojoawo, S.O., Rao, C.V. y Goveas, L.C. (2016). Bioremediation of $\mathrm{Zn}, \mathrm{Cu}$, $\mathrm{Mg}$ and $\mathrm{Pb}$ in Fresh Domestic sewage by Brevibacterium sp. International Journal of Environmental Research, 10(1), 139-148. Recuperado de https://ijer.ut.ac.ir/article_56896_ d 7732 a a 160 e ec 0 a a ab 0 d 6 c 5 ef32e698c.pdf

Organización Mundial de la Salud (OMS) (2011). Adverse health effects of heavy metals in children. Recuperado de https://www.who.int/ceh/capacity/ heavy_metals.pdf

Petrović, M., Šoštarić, T., Stojanović, M., Milojković, J., Mihajlović, M., Stanojević, M. y Stanković, S. (2016). Removal of $\mathrm{Pb} 2+$ ions by raw corn silk (Zea mays L.) as a novel biosorbent. Journal of the Taiwan Institute of Chemical Engineers, 58, 407-416. DOI: https:/doi. org/10.1016/j.jtice.2015.06.025

Poonam, Bharti, S.K. y Kumar, N. (2018). Kinetic study of lead $(\mathrm{Pb} 2+)$ removal from battery manufacturing wastewater using bagasse biochar as biosorbent. Applied Water Science, 8(4), 1-13. DOI: 1https://doi.org/10.1007/s13201018-0765-Z

Ramírez Franco, J.H. y Enríquez Enríquez, M.K. (2015). Remoción de plomo (II) usando lignina obtenida a partir del procesamiento del seudotallo de plátano. Acta Agronómica, 64(3), 209213. DOI: https://doi.org/10.15446/ acag.v64n3.43488

Servicio de Agua Potable y Alcantarillado de Lima Perú (Sedapal) (2009). Valores máximos admisibles de las descargas de aguas residuales no domésticas. Perú.
Recuperado de: http://www.sedapal. com.pe/documents/10154/fedf84051bc2-428e-9d8d-a1c2ad009f53

Sun-Kou, M.R. y Obregón-Valencia, D., Pinedo-Flores, Á., Paredes-Doig, A.L. y Aylas-Orejón, J. (2014). Adsorción de metales pesados empleando carbones activados preparados a partir de semillas de aguaje. Revista de la Sociedad Química del Perú, 80(4), 225-236. Recuperado de https://www.redalyc.org/ articulo.oa? id=3719/371937640002 https://doi.org/10.37761/rsqp. v80i4.159

Tejada, C., Ruiz, E., Gallo, J. y Moscote, J. (2015). Evaluación de la biosorción con bagazo de palma africana para la eliminación de $\mathrm{Pb}$ (II) en solución. Prospectiva, 13(1), 59-67. DOI: https://doi.org/10.15665/rp. v13i1.360

Tejada, C., Villabona, A., Acevedo, D., Pajaro, N. y Otero, M. (2018). Lead (II) remotion in solution using lemon peel (Citrus limonum) modified with citric acid. International Journal of Engineering and Technology (IJET), 10(1), 117-122. Recuperado de www.enggjournals.com/ijet/docs/IJET18-1001-046.pdf https://doi.org/10.21817/ ijet/2018/v10i1/181001046

Tejada-Tovar, C., Gallo-Mercado, J., Moscote, J., Villabona, A. y Acevedo-Correa, D. (2018). Adsorción competitiva de plomo y níquel sobre cáscara de ñame y bagazo de palma en sistema continúo. Biotecnología en el Sector Agropecuario y Agroindustrial, 16(1), 52-61. DOI: 10.18684/bsaa.v16n1.624. https://doi. org/10.18684/BSAA(16)52-61 
United Nations Environment Programme (UNEP) (1999). Environmental impacts of trade liberalization and policies for the sustainable management of natural resources. Uganda.

U.S. Environmental Protection Agency (2014). Global effluent guidelines. Estados Unidos. Recuperado de http://www.levistrauss.com/wp-content/uploads/2014/01/Global-Effluent-Guidelines.pdf

Vizcaíno Mendoza, L. y Fuentes Molina, N. (2015). Biosorción de Cd, Pb y Zn por biomasa pretartada de algas rojas, cáscara de naranja y tuna. Ciencia e Ingeniería Neogranadina, 25(1), 43-60. Recuperado de http://www.scielo.org.co/ pdf/cein/v25n1/v25n1a04.pdf https:// doi.org/10.18359/rcin.432

Wen, X., Du, C., Zeng, G., Huang, D., Zhang, J., Yin, L., ... Wan, J. (2018). Un nuevo biosorbente preparado por Bacillus licheniformis inmovilizado para la eliminación de plomo de las aguas residuales. Chemosphere, 200, 173179. DOI: https://doi.org/10.1016/j. chemosphere.2018.02.078
Wendling, Z.A., Emerson, J.W., Esty, D.C., Levy, M.A., De Sherbinin, A. et al. (2018). Índice de desempeño ambiental 2018. New Haven, CT: Yale Center for Environmental Law \& Policy.

Ye, J., Xiao, H., Xiao, B., Xu, W., Gao, L. y Lin, G. (2015). Biorremediación de solución acuosa contaminada de metales pesados mediante el uso de algas rojas Porphyra leucosticta. Ciencia y Tecnología del Agua, 72(9), 16621666. DOI: 10.2166/wst.2015.386 https://doi.org/10.2166/wst.2015.386

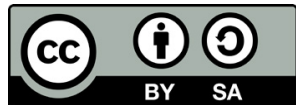

